\title{
Phase Impact Factor: A Novel Parameter for Determining Optimal CT Phase in 4D Radiation Therapy Treatment Planning for Mobile Lung Cancer
}

\author{
Yulin Song*a and Xiaolei Huang ${ }^{\mathrm{b}}$, Boris Mueller ${ }^{\mathrm{c}}$, and Borys Mychalczak ${ }^{\mathrm{c}}$ \\ ${ }^{a}$ Department of Medical Physics, Memorial Sloan-Kettering Cancer Center \\ 1275 York Ave, New York, NY 10021, USA \\ ${ }^{b}$ Department of Computer Science and Engineering, Lehigh University \\ Bethlehem, PA 18015, U.S.A. \\ ${ }^{\mathrm{c}}$ Department of Radiation Oncology, Memorial Sloan-Kettering Cancer Center \\ 1275 York Ave, New York, NY 10021, USA
}

\begin{abstract}
Due to respiratory motion, lung tumor can move up to several centimeters. If respiratory motion is not carefully considered during the radiation treatment planning, the highly conformal dose distribution with steep gradients could miss the target. To address this issue, the common strategy is to add a population-derived safety margin to the gross tumor volume (GTV). However, during a free breathing CT simulation, the images could be acquired at any phase of a breathing cycle. With such a generalized uniform margin, the planning target volume (PTV) may either include more normal lung tissue than required or miss the GTV at certain phases of a breathing cycle. Recently, respiration correlated CT (4DCT) has been developed and implemented. With 4DCT, it is now possible to trace the tumor 3D trajectories during a breathing cycle and to define the tumor volume as the union of these 3D trajectories. The tumor volume defined in this way is called the internal target volume (ITV). In this study, we introduced a novel parameter, the phase impact factor (PIF), to determine the optimal CT phase for intensity modulated radiation therapy (IMRT) treatment planning for lung cancer. A minimum PIF yields a minimum probability for the GTV to move out of the ITV during the course of an IMRT treatment, providing a minimum probability of a geometric miss. Once the CT images with the optimal phase were determined, an IMRT plan with three to five co-planner beams was computed and optimized using the inverse treatment planning technique.
\end{abstract}

Keywords: Lung cancer, ITV, IMRT, 4DRT, PIF, MIP, $\mathrm{f}_{\text {dam }}$, Bellows system

* songy@mskcc.org; Phone: 1-914-333-8676; Fax: 1-914-366-4749; www.mskcc.org 


\section{INTRODUCTION}

Despite all the efforts, lung cancer is still the leading cause of cancer related deaths in the United States. About $80 \%$ of all primary lung cancers are non-small cell lung carcinomas (NSCLC) ${ }^{[1]}$. At present time, the optimal curative treatment for patients with primary early-stage NSCLC without distant metastasis is complete surgical resection ${ }^{[2]}$. Unfortunately, only a quarter of all patients have this valuable option. For patients with early stage (T1-T2) medically inoperable disease, radiation therapy is the only treatment of choice. For locally advanced (T3) NSCLC, especially those cases involving the mediastinum, concurrent radiation therapy and chemotherapy (radio-chemotherapy) are the preferred treatment modality. Despite significant technological progress has been made in radiation oncology in recent years, 5-year survival rate remains at about $15 \%$ in patients with early stage NSCLC ${ }^{[2]}$. Although dose escalation can potentially improve survival, it is constrained by radiation pneumonitis. Clinical investigations have shown that the incidence of pneumonitis is correlated not only to dose, but also to the irradiated lung volume. It has been reported that when the lung volume receiving more than $20 \mathrm{~Gy}\left(\mathrm{~V}_{20}\right)$ exceeds $30 \%$, i.e., $\mathrm{V}_{20}>30 \%$, the risk of radiationinduced pneumonitis is greater than $10 \%{ }^{[3-4]}$. Local control can be improved by more accurately defining the tumor volume, including both macroscopic and microscopic components, precisely delivering higher doses to PTV with higher fractionations, and better controlling internal organ motion due to respiration.

The complexity in treating lung cancer using radiation therapy is that the tumor is not stationary in lung due to respiratory motion. Clinical measurements using target tracking or image-guided techniques have shown that tumor can move significantly during a free breathing cycle. Depending on tumor location, the tumor could move from a few millimeters to several centimeters. If respiratory motion is not carefully modeled during the treatment planning phase, the highly conformal dose distribution with steep gradients created by intensity modulated radiation therapy (IMRT) could miss the target. If dose escalation is to be used for the treatment, this could result in radiation pneumonitis and esophagitise, leading to an increased risk of complications. To account for this periodical respiratory motion and avoid target miss, the most common approach is to add a population-derived safety margin to the gross tumor volume (GTV). However, such a one-size-fits-all approach neglects the basic fact that respiratory motion is not isotropic and is patient-dependent. It is not tailored to each individual motion characteristics. In addition, during a free breathing CT simulation, the images could be acquired at any phase of a breathing cycle. This is particularly the case for the common single-slice CT scanners. Low rotation rate produces images with poor interslice correlation. With such a generalized uniform margin, the planning target volume (PTV) may either include more normal lung tissue than required or miss the GTV at certain phases of a breathing cycle.

Another common solution to respiratory motion is respiratory gating using an infrared sensor placed on the patient chest surface. Radiation beam will be turned on when patient breath enters a predetermined phase window. This is usually chosen to be at end-exhalation where the breath tends to stay slightly longer than at end-inhalation. In addition, the analysis of breathing pattern has shown that cough usually occurs right after end-inhalation, producing severe breathing irregularities. Therefore, the end-exhalation phase is more reproducible and stable. However, studies have also shown that the movement of the PTV does not necessarily follow the sinusoidal movement of the external sensor. For some patients, the anatomic motion can be out of phase with the external sensor motion. This is not surprising because the external sensor only samples one point on the truck and does not represent the global picture of the lung ventilation. In addition, if the effective duty cycle, defined as the ratio of total beam-on time to the time needed to complete a treatment, is $1 / 4$, the total 
treatment time will be increased by 3 times. For very busy cancer centers, this is certainly the biggest bottle-neck for implanting this technology.

Recently, respiration correlated CT (4DCT) has been developed and implemented in radiation oncology. The time information required for 4D imaging can be obtained prospectively or retrospectively using respiratory motion gating or tracking techniques. With 4DCT, it is now possible to trace the tumor $3 \mathrm{D}$ trajectories during a breathing cycle using a technique called the maximum intensity projection (MIP) and to define the tumor volume as the union of these 3D trajectories. The tumor volume defined in this way is called the internal target volume (ITV). In this paper, we introduced a novel parameter, the phase impact factor (PIF), to determine the optimal CT phase for IMRT treatment planning for lung cancer. A minimum PIF yields a minimum probability for the GTV to move out of the ITV during the course of an IMRT treatment, providing a minimum probability of a geometric miss.

\section{MATERIALS AND METHODS}

\subsection{Respiratory tracking system}

At MSKCC regional cancer centers, we use the Bellows tracking system to track the respiratory motion. The Bellows tracking system is an elastic air coil embedded with a pressure transducer, as shown in Figure 1(a). The system is integrated with the CT scanner's data acquisition and reconstruction systems. Prior to scanning, it is attached to the patient at the site of diaphragm Figure 1(b). It expands and contracts in synchronization with respiratory motion. The pressure transducer converts the periodical pressure waveform into a voltage signal, which is then digitized and transmitted to the CT scanner for retrospective data sorting and multiphase reconstruction.
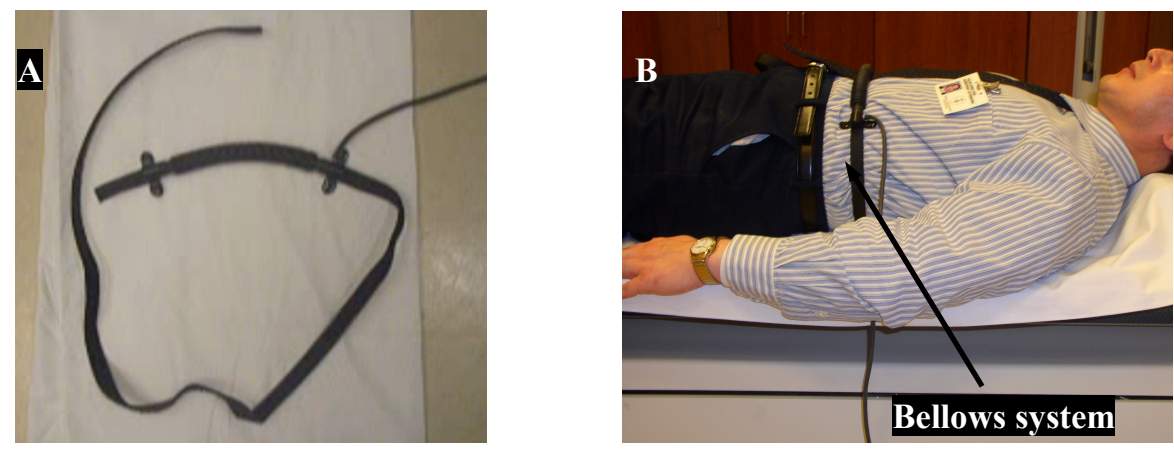

Figure 1. The Bellows tracking system (A) and the placement of the system on a patient (B)

\subsection{Respiration correlated CT scan}

Respiration correlated CT scan is performed using the Brilliance Big Bore CT scanner (Philips Medical Systems, Cleveland, OH, USA). The scanner has an $85 \mathrm{~cm}$ bore, providing a maximum of $60 \mathrm{~cm}$ field of view (FOV). The detector width (collimation) is $24 \mathrm{~mm}$ (16 x 1.5). A rotation speed of 0.5 second is used for routine 4D simulation. The maximum allowed pitch is determined by formula: Rotation Time ( $\mathrm{sec})$ x Breath Rate (breaths/min) / 60 ( $\mathrm{sec} / \mathrm{min})$. A pulmonary 4D retrospective spiral protocol is used for the scan. Once a steady pulmonary waveform has been 
established, the scan is trigged. The scan is acquired at a very low pitch (low table speed) so that any scanned voxel remains within the detector width throughout a complete breathing cycle. The oversampled projections with timestamps provide enough phase information for the post-retrospective multiphase reconstruction. The scan time is about $90 \sim 110$ seconds for a typical lung simulation. The patient is scanned in a supine position in a customized thermoplastic mold to minimize patient movement during the data acquisition. The scan is acquired from the thoracic inlet to the costophrenic angles. During the scan, the patient is instructed to breath in a normal shallow pattern to reduce artifacts in the reconstructed multiphase images.

\subsection{Retrospective multiphase reconstruction}

With these 4D projections, a 4D image data set, consisting of a series of 3D images at user-selected phases can be reconstructed retrospectively. Each of these 3D images has the same phase and thus, appears motion-free. Retrospective Multiphase Reconstruction (RMR) is based on a sorting technique. Prior to reconstruction, 4D projections at all respiratory phases are sorted into appropriate phase bins. Projections with the same phase are grouped together, suppressing motion blur from other phases. Then, a series of motion-free 3D images corresponding to different phases of the breathing cycle are reconstructed using the filtered-back projection algorithm Because of breathing irregularities, especially for lung cancer patients, it was found that sorting by respiratory amplitude is superior to respiratory phase sorting. In our practice, since 4DCT image is acquired only after a steady pulmonary waveform has been established, we use respiratory phase sorting for multi-phase image reconstruction. On this basis, a retrospective eleven-phase reconstruction will then be performed. The phases are equally spaced, ranging from $0,10,20,30,4050,60,70,80,90$, and $100 \%$ of a normal breathing cycle. We define $0 \%$ as end-inhalation and $50 \%$ as end-exhalation, $25 \%$ as mid-exhalation, and $75 \%$ as mid-inhalation (Figure 2 ).

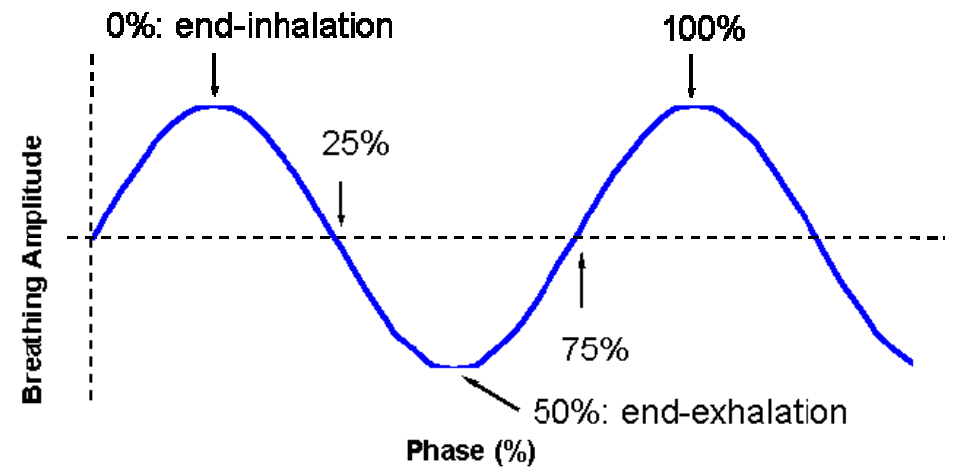

Figure 2. Schematic diagram of phase definition for an eleven-phase retrospective reconstruction

\subsection{Maximum intensity Projection}

After the retrospective multiphase reconstruction is completed, a series of maximum intensity projection (MIP) images is created. The MIP algorithm uses a series of parallel rays projected through a stack of CT images having the same spatial relationship. An MIP image is constructed by assigning each pixel the highest CT number of all slices along the same projection ray, with all other CT numbers being discarded. Thus, more than $95 \%$ of the original 3D data are lost. A series of MIP images provides a new 3D CT dataset. Each of the voxels has the highest intensity value throughout 
the 4D CT dataset, providing a full intensity display of the brightest object. In pulmonary imaging, the MIP images can accurately trace the trajectory of a moving lung tumor with high intensity and contrast during a respiratory cycle.

\subsection{Internal target volume}

After MIP images are created, ITV is then delineated slice by slice on MIP images by the radiation oncologist using Tumor Localization, a volume drawing utility on the CT console. The ITV delineated on MIP images is essentially the union of the individual gross tumor volumes (GTV) defined at selected phases of a breathing cycle. Thus, under a perfect patient setup condition, the GTV would always move within a space confined by the ITV.

\subsection{Phase impact factor}

Intuitively, there should exit a CT data set at a certain phase where the GTV is optimally located in the ITV. The optimal location means that at this specific phase, the margins or separations between the GTV and the ITV are the largest. These margins distribute most uniformly so that the GTV has the minimum probability of moving out of the ITV during the course of a radiation treatment, providing a minimum probability of a target miss. Assuming that patient setup uncertainties prior to treatment are completely random and homogeneous, we introduce a novel parameter called the Phase Impact Factor (PIF) to quantitatively search for such an optimal phase during a free breathing CT simulation for 4D radiation therapy (4DRT). Firstly, we calculate the mean margin between the GTV and ITV. We then calculate the variance of the margin. The PIF is defined as:

$$
\mathrm{PIF}=\frac{\text { Variance of Margin }}{\text { Mean Margin }}
$$

We now seek for a phase among all different phases that yields a minimum PIF, which corresponds to the minimum probability of the GTV moving beyond the ITV. Once the CT data set with the optimal phase is determined, an IMRT plan is computed and optimized using the inverse treatment planning technique.

\subsection{IMRT treatment planning}

IMRT treatment planning is performed on an MSKCC in-house treatment planning system. The planning target volume (PTV) is created by adding an appropriate setup uncertainty margin to the ITV by the radiation oncologist. Depending on the location of GTV, a non-uniform margin may be necessary to maximum spare the lungs. To achieve a better target dose coverage, an expanded PTV, named as PTVE, is created by adding a uniform $3 \mathrm{~mm}$ margin to the PTV. In addition, the planner also copies it above and below the PTV by 2 slices to avoid cold spots in these regions. The organs at risk (OAR) are the cord, esophagus, heart, and lungs, named as CORD, ESOPH, and HEART, and LUNGS. The heart and esophagus are delineated by the radiation oncologist and the cord and lungs by the treatment planner. To better spare the cord and esophagus, a $3 \mathrm{~mm}$ uniform margin is added to CORD and ESOPH to create CORDE and ESOPHE. Additionally, two tuning structures, INRIND and OUTRIND, are also created for dose tuning purpose. INRIND is created by adding a $6 \mathrm{~mm}$ uniform margin to the PTVE. OUTRIND is generated by adding a $40 \mathrm{~mm}$ or more uniform margin to the PTVE. By assigning proper dose constraints to INRIND and OUTRIND, they could be used to fine tune the dose outside the PTV and thus, to eliminate the undesirable hot spots in these areas. 
Each IMRT plan consists of three to five co-planner beams. The beam angles are approximately equally spaced between anterior-posterior (AP) and posterior-anterior (PA) directions. Depending on the location of the PTV, non-equally spaced beams are also used to better spare the heart and esophagus. More beams would certainly produce a more conformal target dose distribution. However, they would also deliver unnecessary low dose to a larger lung volume. To spare the contra-lateral lung, all beams enter the target through the involved lung only. Optimization-only structures include PTVE, CORDE, LUNGS, ESOPHE, HEART, and RIND defined as the logical operation: OUTRIND-INRIND. The plan evaluation structures are PTV, CORD, ESOPH, HEART, and LUNGS.

Given a set of dose limits and dose-volume constraints to the optimization-only structures, the IMRT plan is optimized by minimizing a quadratic objective function using an iterative gradient search algorithm. The objective function consists of terms corresponding to the PTV and the organs at risk. The goal of IMRT optimization is to find a set of ray intensities or an intensity map that minimizes the objective function. Although the inverse planning optimization is performed automatically on a high speed personal computer (PC), the adjustment of various constraints is done interactively and manually through a trial and error process. Since the constraints don't have an intuitive and linear relationship with the desired dose distribution, it could take numerous trials before finding a set of constraints that result in a desirable dose distribution. After many runs, if the criteria for plan acceptance are still not met, a trade-off between the target dose coverage and constraints would have to be made. Once optimal intensity maps are obtained, leaf sequences are generated using the dynamic MLC (DMLC) technique. Based on these leaf sequences, the final dose distribution is then computed using a pencil beam algorithm. In addition, we also compute the normal tissue complication probability (NTCP) and fractional lung units damaged $\left(f_{\text {dam }}\right)$ for our dose escalation protocol. We push the dose to as high as $8100 \mathrm{cGy}$ if NTCP is less than or equal to $0.15, f_{\text {dam }}$ is less than or equal to 0.27 , and the mean lung dose is less than $2100 \mathrm{cGy}$. In addition, we also use $\mathrm{V}_{20}<$ $30 \%$ as one of our plan acceptance criteria.

\section{RESULTS}

\subsection{Phase impact factor}
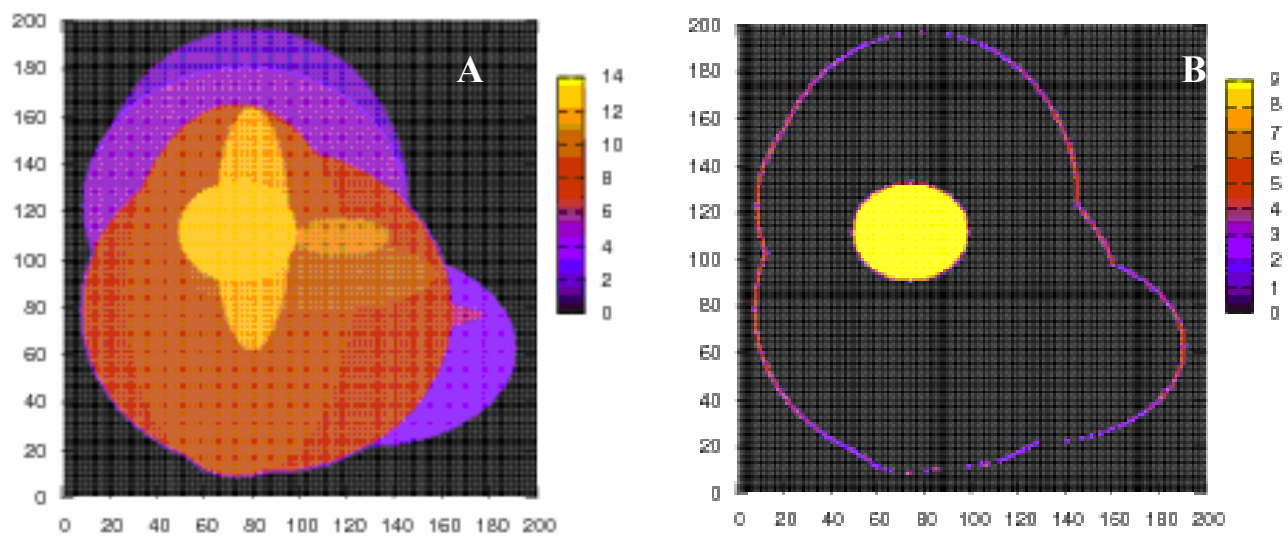

Figure 3. ITV (the envelope of ellipses), GTV (ellipses) (A), and the optimal ellipse determined by the minimum PIF (B). 
To verify that a minimum PIF indeed provides a CT data set with desired properties, we performed a series of simulations. Many ellipses, representing various GTVs in different phases and breathing conditions, were generated with random centers and axes. Figure 3 shows the ITV (shaded area) and GTVs (ellipses). Their corresponding mean margin and the variance of the margin at each phase were computed. From a minimum PIF, the corresponding location of the ellipse was determined as the optimal phase. It was found that the ellipse with the minimum PIF met our requirements. We also changed the random seed number. A series of new ellipses was then generated and these processes were repeated until a pre-determined run number was reached. The simulations indicated that the PIF gave consistent results and was a reliable planning parameter

\subsection{ITV and PTV}

Figure 4 shows the GTV, ITV, and PTV for a representative lung case. In this particular example, the margin between the GTV and ITV is the largest in the anterior direction, indicating that the GTV moves in this direction during a free breathing cycle. The pink, brown, and red solid lines represent the GTV, ITV, and PTV, respectively.

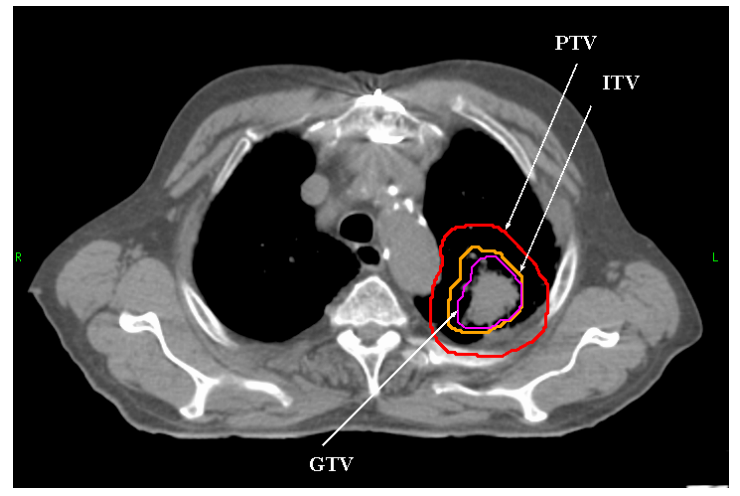

Figure 4. The delineation of GTV, ITV, and PTV for a representative lung case. A necessary margin was added to ITV to create PTV to account for the daily patient setup uncertainty. The patient was in a supine position.

\subsection{IMRT plan}

Figure 5 shows the isodose distribution of a coronal slice for a lung cancer patient. The yellow and red dotted lines represent the ITV and PTV, respectively. The patient was an 80 year old male with a $4.0 \times 2.6 \mathrm{~cm}$ spiculated left upper lobe mass. A PET/CT scan confirmed a $3.6 \mathrm{~cm}$ left upper lobe lesion with an elevated standard uptake value (SUV) of 8.9. Biopsy showed that this was a squamous cell carcinoma. Due to poor pulmonary function, the patient was not considered to be an adequate candidate for surgery. Therefore, the patient received a definitive IMRT treatment to $80 \mathrm{~Gy}$, delivered in 2 Gy per fraction. A three-field IMRT plan was computed for the patient, with gantry angles of $0^{\circ}$,

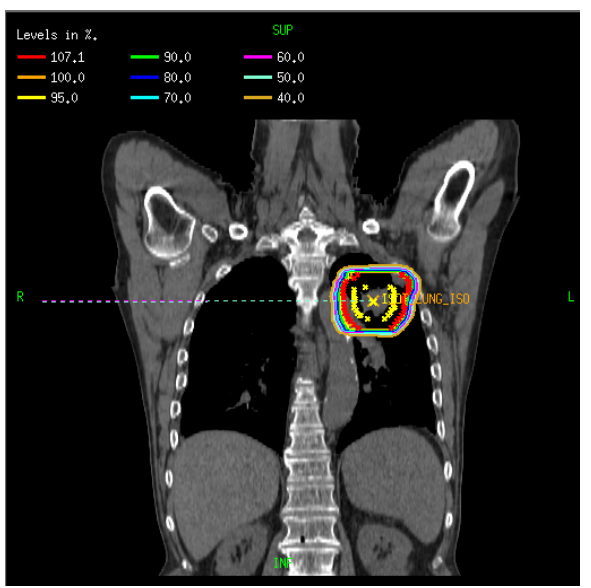

Figure 5. The isodose distribution of a typical coronal slice for a lung cancer IMRT plan 
$200^{\circ}$, and $340^{\circ}$. All beams entered the target through the involved side only to spare the right lung. The PTV was $198.5 \mathrm{~cm}^{3}$. As indicated in Figure 5, the brown solid line represents $100 \%$ isodose line, covering the PTV tightly. Even with a three-beam arrangement, the minimum PTV dose was 96.91\%, while the maximum PTV dose was $108 \%$, showing reasonably good homogeneous dose distribution. The mean PTV dose was $103.67 \%$. $\mathrm{D}_{95}$ and $\mathrm{V}_{95}$ were $101.3 \%$ and $100 \%$, respectively. The maximum cord dose was 7.8\%. The maximum heart and esophagus doses were $4.38 \%$ and $14.0 \%$, respectively. The mean heart and esophagus doses were $0.4 \%$ and $4.7 \%$, which was, in part, due to three beams being used in the IMRT plan. The maximum right lung dose was $16.2 \%$ and the mean right lung was $0.2 \%$. The maximum left lung dose was $108.4 \%$ and the mean dose was $29.9 \%$. This was expected as the tumor was in the left lung. $\mathrm{V}_{20}$ was $14.6 \%$, which was computed based on combined total lung volume. The $\mathrm{V}_{20}$ was much lower than the commonly accepted $30 \%$. Since the dose escalation protocol was used in this case, we also computed NTCP and $f_{\text {dam }}$, which were 0.01 and 0.14 , respectively, well below our established plan acceptance criteria. Figure 6 shows the dosevolume histogram (DVH) for this plan. Figure 7 is a DRR showing the beam aperture that was projected at the gantry angle of $200^{\circ}$ (right-posterior). The red and yellow solid lines are the PTV and ITV, respectively. The horizontal white parallel lines are MLC leaves used for beam shaping and intensity modulation.

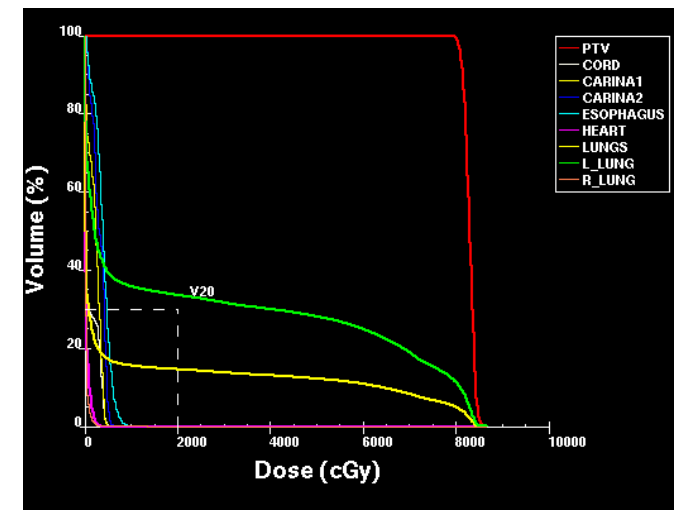

Figure 6. The dose-volume histogram of the IMRT plan. The red line represents PTV.

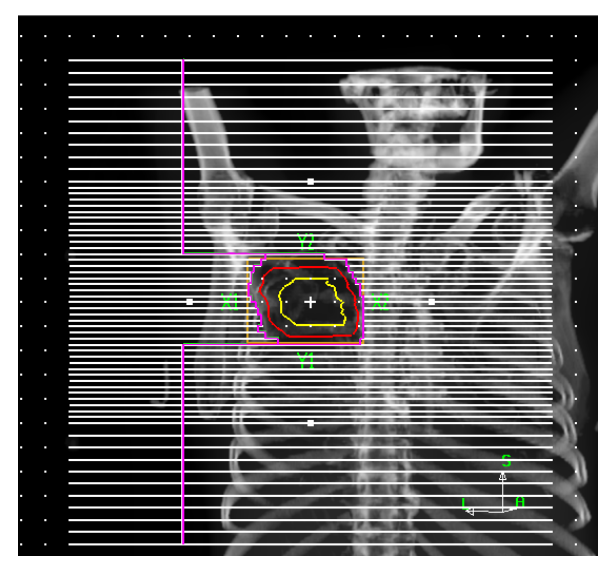

Figure 7. A DRR projected at the gantry angle of $200^{\circ}$, showing the beam aperture at this direction. 


\section{CONCLUSIONS}

We believe that the phase impact factor introduced in this study could be used as the most conservative phase selection criterion in 4D radiation therapy for lung cancer. Since PIF is site independent, it can also be used on other tumor sites that are affected by respiration, such as the stomach and liver. The definition of the PIF is one of the many possible mathematical models. The PIF could offer a safer CT data set than the most commonly used phase-averaged CT images. In addition, our initial experience with the Bellows tracking system indicated that the Bellows system is easy to use and provides a practical and quick solution to 4D RT for a busy radiation oncology department. We believe that the Bellows tracking system is a competitive technology and will continue to co-exit with the respiratory gating technology.

\section{REFERENCES}

[1] Cancer Facts and Figures 2005, American Cancer Society.

[2] Nesbitt, J.C., Putnam, Jr. J.B., Walsh, G.L., Roth, J.A., Mountain, C.F., Walsh GL, Roth JA, and Mountain, C.F. "Survival in early-stage non-small cell lung cancer." Ann Thorac Surg 60:466-72 (1995).

[3] Seppenwoolde, Y., Lebesque, L.W., de Jaeger, K., et al. "Comparing different NTCP models that predict the incidence of radiation pneumonitis." Int $J$ Radiat Oncol Biol Phys 55:724-35 (2003).

[4] Graham, M.V., Purdy, J.A., Emami, B., et al. "Clinical dose-volume histogram analysis for pneumonitis after 3D treatment for nonsmall cell lung cancer." Int J RadiatOncol Biol Phys 45:223-9 (1999). 